\title{
Use of adenosine deaminase as a diagnostic tool for tuberculous pleurisy
}

\author{
Lesley J Burgess, Frans J Maritz, Irene Le Roux, J J Frans Taljaard
}

\begin{abstract}
Background - A statistical audit of adenosine deaminase (ADA) in pleural effusions was undertaken.

Methods - ADA analysis, cytological and microbiological examinations, and differential cell counts were performed on 462 pleural fluid samples.

Results - ADA activity in tuberculous effusions was higher than in any other diagnostic group. At a level of $50 \mathrm{U} / 1$ the sensitivity and specificity for the identification of tuberculosis was $90 \%$ and $89 \%$, respectively.

Conclusions - ADA activity remains a useful test in the evaluation of pleural effusions.
\end{abstract}

(Thorax 1995;50:672-674)

Keywords: adenosine deaminase, tuberculosis, pleural effusion.

The increased incidence of tuberculosis in South Africa (172 per 100 000), and in particular in the Western Cape (670 per 100 000), ${ }^{1}$ necessitates the adoption of a reliable, rapid, diagnostic test. Diagnostic tools for tuberculous pleurisy include pleural fluid cultures (sensitivity $20-30 \%$ ), ${ }^{2}$ pleural biopsies (sensitivity $50-80 \%),{ }^{3}$ polymerase chain reaction (sensitivity $78 \%$ for active disease), ${ }^{4}$ and pleural fluid levels of adenosine deaminase (ADA)..$^{5-11}$

Serious doubt has been cast on the usefulness of ADA levels in the detection of tuberculous pleurisy. ${ }^{1213}$ We therefore set out to perform a statistically acceptable audit on the determination of $\mathrm{ADA}$ in pleural effusions.

\section{Methods}

A study was carried out at Tygerberg Hospital during 1993 and included all patients referred for thoracentesis. ADA activity (U/l) was determined according to the method described by Giusti. ${ }^{14}$ Microbiological and cytological examinations and differential cell counts were also performed.

Patients were classified into the following groups based on predetermined criteria ${ }^{15}$ : tuberculosis $(n=154)$, malignancy $(n=68)$, infection $(n=64)$, miscellaneous exudates $(n=$ $20)$, congestive cardiac failure $(n=72)$, and other transudates $(n=35)$. Patients with multiple superimposed diseases or effusions of unknown origin were classified as "undiagnosed" $(n=30)$. Patients with empyemas and haemothoraces were excluded.

The ADA activity in tuberculous effusions was compared with that in the other diagnostic groups by the Wilcoxon two sample test. Data that were not normally distributed are given as median (25th, 75th percentile) values. The use of ADA as a diagnostic tool for tuberculosis was evaluated at various cutoff levels by calculating sensitivity, specificity, and positive (PPV) and negative (NPV) predictive values. These were compared by means of relative operating characteristic curves. ${ }^{16}$

Results

Although 462 specimens were received for ADA analysis, 19 patients were excluded because of a haemothorax $(n=5)$ or empyema $(n=14)$. The remaining 443 patients comprised 294 patients of mixed race, 89 black patients, and 60 Caucasians, of which 245 were men and 198 women. The mean (SD) age of the patient population was 46 (21) years (range 6 months to 98 years).

The following diagnostic criteria were required for the patients with tuberculous pleuritis: (a) identification of the bacillus in the pleural fluid or biopsy specimen by stain or culture, or by the presence of granulomas in the biopsy tissue $(n=87$, of which six had smear positive and 25 had culture positive sputums); or (b) positive sputum culture in the presence of clinical and radiological evidence for tuberculosis $(n=31$, of which eight had smear positive sputums); or (c) clinical and radiological evidence of tuberculosis associated with a response to antituberculosis therapy $(n=$ 36).

The distribution of ADA activity for the tuberculosis subclasses is shown in fig 1 . No significant difference was found in ADA activities between the tuberculosis subclasses. The distribution of ADA levels in the various diagnostic groups is shown in fig 2 . The median (25th, 75th percentile) values for patients with tuberculosis, infective and malignant effusions were $102(71,141) U / 1,29(20,61) U / 1$, and $25(15,34) U / 1$, respectively. ADA activity was significantly higher for tuberculosis than for 


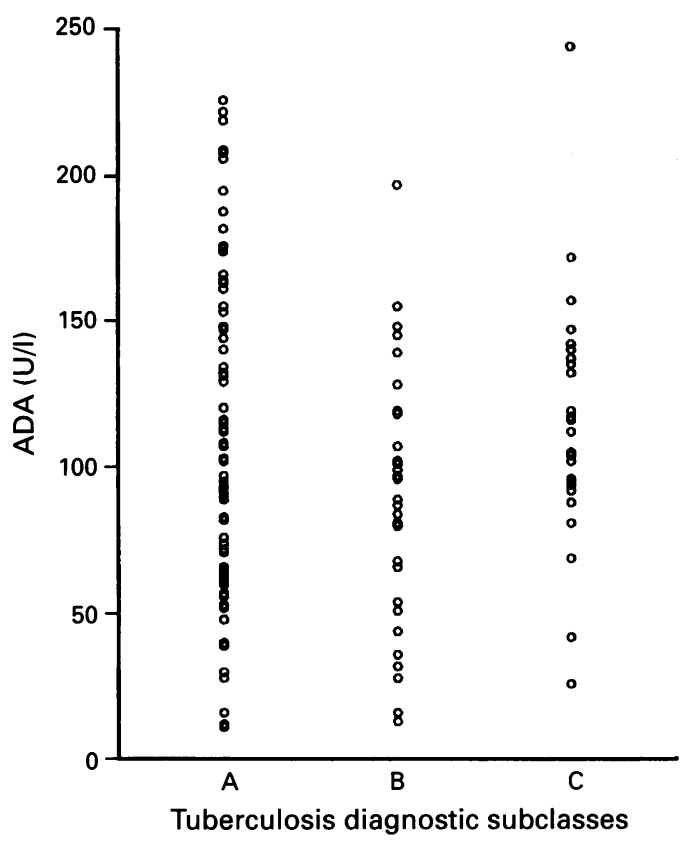

Figure 1 Distribution of $A D A$ activity in the tuberculosis diagnostic subclasses. $A=$ identification of the bacillus in pleural fluid or biopsy specimen or by the presence of granulomas in biopsy tissue (median (25th, 75th percentile) values $103(69,149)$ U/l); $B=$ positive sputum culture in the presence of clinical and radiological evidence for tuberculosis $(90(55,117) U / l)$; and $C=$ clinical and radiological evidence for tuberculosis associated with a response to antituberculous therapy $(113(96,136)$ $U / l)$.

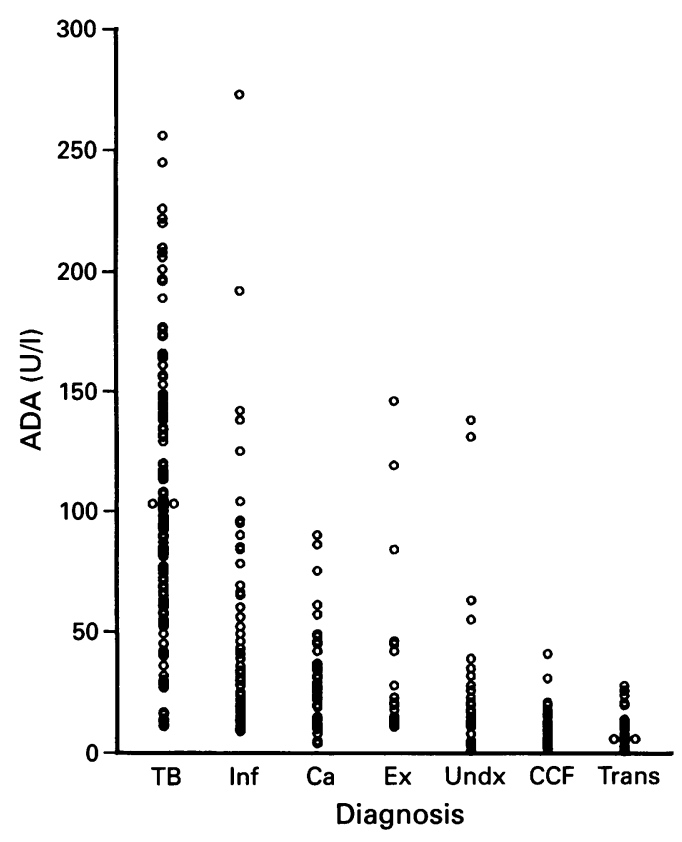

Figure 2 Distribution of $A D A$ activity in the various diagnostic subclasses. $T B=$ tuberculosis (median (25th, 75th percentile) values $102(71,141)$ U/l); Inf = infective (29 (20, 61) U/l); Ca=malignancy $(25(15,34) U / l)$; Ex= miscellaneous exudates $(21(15,43)$ U/l); Undx= undiagnosed $(18(12,34) U / l) ; C C F=$ congestive cardiac failure $(9(6,12)$ U/l); Trans = miscellaneous transudates $(8(6,13) U / l)$.

the other diagnostic groups $(p<0.005$ for each group).

Various levels of ADA were tested as a cutoff level for the diagnosis of tuberculosis; $50 \mathrm{U} / 1$ was found to yield the best results. This corresponded to a sensitivity, specificity, PPV, and NPV of $90 \%, 89 \%, 81 \%$, and $94 \%$, respectively. There were 32 "false positive" cases, which included 17 infective effusions (13 having relative neutrophilia and one having a relative lymphocytic predominance; no differential cell counts were obtained in the remaining three cases); five malignant effusions (three being due to haematological malignancies); four miscellaneous exudates (chylothorax, systemic lupus erythematosis, asbestosis, and a ruptured hydatid cyst); and six undiagnosed effusions.

\section{Discussion}

ADA (EC 3.5.4.4), a polymorphic enzyme involved in purine metabolism, catalyses the deamination of adenosine to inosine and ammonium. ${ }^{17}$ Although found in most tissues, ADA activity is greatest in the lymphoid tissues, ${ }^{17}$ its activity being $10-20$ times more active in $T$ lymphocytes than in B lymphocytes. $^{18}$ ADA plays a part in the differentiation of lymphoid cells ${ }^{18}$ and the maturation of monocytes to macrophages. ${ }^{19}$ In pleural fluids it reflects the cellular immune response in the pleural compartment, especially the activation of T lymphocytes.

Several studies have shown the diagnostic use of ADA in tuberculous pleurisy. ${ }^{5-11}$ Our study supports this evidence. Two previous studies, however, have shown that ADA levels are of limited value; one was performed in an area of low tuberculosis prevalence, ${ }^{12}$ and the other included poorly defined population groups. ${ }^{13}$

In this study $\mathrm{ADA}$ activity was highest among the tuberculosis group. Although infective conditions may also be associated with high ADA activities, the relative cell count can be used to distinguish between these two entities. Tuberculous effusions are usually characterised by a relative lymphocytosis, and infective effusions by a neutrophil predominance. ${ }^{20}$ High lymphocyte counts can also be found in effusions secondary to connective tissue disorders and malignancies, ${ }^{2021}$ particularly those secondary to haematological malignancies.

Previous studies have found increased ADA levels in empyemas, ${ }^{10}$ where the immune response involves polymorphonuclear cells and macrophages rather than lymphocytes. The high ADA activity thus results chiefly from large phagocytic populations. ${ }^{810}$ Because of interference resulting from their turbidity, empyemas were excluded from our study.

ADA activity is useful for diagnosing tuberculous pleuritis and values ranging from 40 to $70 \mathrm{U} / 1$ have been advocated as cutoff levels. ${ }^{5-11}$ Based on relative operating characteristic curves, the best results are yielded at $50 \mathrm{U} / 1$ which corresponds to a sensitivity, specificity, PPV, and NPV of $90 \%, 89 \%, 81 \%$, and $94 \%$, respectively.

Tuberculous pleurisy is traditionally diagnosed by identification of Mycobacterium tuberculosis in pleural fluid or biopsy specimen. Long culture periods often result in clinical and therapeutic decisions being made before these 
laboratory results become available. ${ }^{4}$ The use of pleural fluid levels of ADA provides a rapid and accurate method of suggesting a diagnosis of tuberculosis, especially in high prevalence areas, thereby expediting the initial decision making process and management of the patient. It is important to recognise, however, that pleural fluid levels of ADA may be high in other conditions, and particularly when the cause is a pyogenic infection.

We would like to extend our sincere gratitude to the Cape Provincial Administration for use of facilities; Dr I J M Venter for assistance with the collection of samples; and Drs J van Greune and ME Carstens for continuous and critical revision of the manuscript.

1 Küstner HGV. Epidemiological comments. Epidemiology of the Department of National Health and Population Development 1993;20:15-6.

2 Berger HW, Mejia E. Tuberculous pleurisy. Chest 1973;63: 88-92.

3 Jay SJ. Diagnostic procedures for pleural diseases. Symposium on pleural diseases. Clin Chest Med 1985;6:33-48.

4 De Wit D, Maartens G, Steyn L. A comparative study of De Wit D, Maartens $G$, Steyn $L$. A comparative study of
the polymerase chain reaction and conventional procedures for the diagnosis of tuberculous pleural effusion. Tubercle 1992;73:262-7.

5 Piras MA, Gakis C, Budroni M, Andreoni G. Adenosine deaminase activity in pleural effusions: an aid to differentia diagnosis. $B M \mathcal{F}$ 1975;3:192-3.

6 Maritz FJ, Malan C, Le Roux I. Adenosine deaminase estimations in the differentiation of pleural effusions. $S$ Afr Med $¥$ 1982;62:556-8.

7 Ocaña I, Martinez-Vazquez JM, Segura RM, Fernandez-DeSevilla T, Capdevila JA. Adenosine deaminase in pleural fluids. Test for diagnosis of tuberculous pleural effusion. Chest 1983;84:51-3.

8 Strankinga WFN, Nauta JJP, Staub JP, Stam J. Adenosine deaminase activity in tuberculous pleural effusions: a diag nostic test. Tubercle 1987;68:137-40.

9 Bañales JL, Pineda PR, Fitzgerald JM, Rubio H, Selman $M$, Salazar-Lezama $M$. Adenosine deaminase in the diag nosis of tuberculous pleural effusions. A report of 218 nosis of tuberculous pleural effusions. A report of 218

10 San José E, Valdés L, Sarandeses A, Alvarez D, Chomon B. Diagnostic value of adenosine deaminase and lysozyme in tuberculous pleurisy. Clin Chim Acta 1992;209:73-81. 11 Valdés L, San José E, Alvarez D, Sarandeses A, Pose A, Chomon B, et al. Diagnosis of tuberculous pleurisy using the biologic parameters adenosine deaminase, lysozym and interferon gamma. Chest 1993;103:458-65.

12 Van Keimpema ARJ, Slaats EH, Wagenaar JMP. Adenosine deaminase, not diagnostic for tuberculous pleurisy. Eur $\mathcal{F}$ Respir Dis 1987;71:15-8.

13 Maartens G, Bateman ED. Tuberculous pleural effusions: increased culture yield with bedside inoculation of pleural fluid and poor diagnostic value of adenosine deaminase. Thorax 1991;46:96-9.

14 Giusti G. Adenosine deaminase. In: Bergmeyer HU, ed Methods of enzymatic analysis. New York: Academic Press 1974:1092-6.

15 Burgess LJ, Maritz FJ, Taljaard JJF. Comparative analysis of the biochemical parameters used to differentiate between pleural exudates and transudates. Chest 1995 (in press)

16 Beck JR, Schultz EK. The use of relative operating characteristic (ROC) curves in test performance evaluation. Arch Pathol Lab Med 1986;110:13-20.

17 Van der Weyden MB, Kelley WN. Human adenosine deaminase distribution and properties. $\mathcal{F}$ Biol Chem 1976 251:5448-56.

18 Barton RW, Goldshneider I. Nucleotide metabolizing enzymes and lymphocytic differentiation. Mol Cell Biochem 1979;28:135-47.

19 Fischer D, van der Weyden MB, Snyderman R, Kelley WN. A role for adenosine deaminase in human monocyte maturation. $\mathcal{F}$ Clin Invest 1976;2:399-407.

20 Sahn SA. State of the art. The pleura. Am Rev Respir Dis 1988;138:184-234.

21 Yam LT. Diagnostic significance of lymphocytes in pleural effusions. Ann Intern Med 1967;66:972-82.

\title{
Posterior subcapsular cataract and inhaled corticosteroid therapy
}

\author{
Faisal Abuekteish, J N P Kirkpatrick, George Russell
}

\section{Department of Medical Paediatrics, Royal Aberdeen Children's Hospital, Foresterhill, Aberdeen AB9 2ZG, UK F Abuekteish G Russell}

\section{Department of Ophthalmology, University of Aberdeen, Foresterhill, Aberdeen AB9 2ZD, UK J N P Kirkpatrick \\ Reprint requests to: Dr G Russell. \\ Received 7 October 1994 Returned to author 5 December 1994 Revised version received 29 December 1994 Accepted for publication 20 January 1995}

\begin{abstract}
Background - Although posterior subcapsular cataract complicates both systemic and topical corticosteroid therapy, the literature on the effects of inhaled corticosteroids is conflicting.

Methods - One hundred and forty children and young adults on inhaled corticosteroids were examined by slit lamp ophthalmoscopy after pupillary dilatation; 103 had received one or more short courses ( $\leqslant 7$ days) of oral corticosteroids in the management of acute asthmatic attacks and four had also received one or more prolonged courses ( $\geqslant 4$ weeks) of alternate day oral corticosteroid therapy.

Results - Bilateral posterior subcapsular cataract was identified in one girl who had received several prolonged courses of oral corticosteroids, but was not identified in any other patient.
\end{abstract}

Conclusions - There is no evidence to support the contention that inhaled corticosteroid therapy on its own, or in association with short courses of oral corticosteroid therapy, might cause cataracts. Although children receiving long term systemic corticosteroid therapy should be screened for cataracts, this is unnecessary in children on inhaled corticosteroids alone.

(Thorax 1995;50:674-676)

Keywords: childhood asthma, posterior subcapsular cataract, corticosteroids.

The association between systemic corticosteroid therapy and the development of posterior subcapsular cataract, first described by Black et al in adults in $1960,{ }^{1}$ has also been reported in children receiving systemic corticosteroids for asthma. There are also reports 